\title{
2D Motion Analysis of Long Axis Cardiac Tagged MRI
}

\author{
Ting Chen, Sohae Chung, and Leon Axel \\ Radiology Department, New York University, School of Medicine \\ 600A 650 First Ave, New York City, NY, 10016, USA
}

\begin{abstract}
The tracking and reconstruction of myocardial motion is critical to the diagnosis and treatment of heart disease. Currently, little has been done for the analysis of motion in long axis (LA) cardiac images. We propose a new fully automated motion reconstruction method for grid- tagged MRI that combines Gabor filters and deformable models. First, we use a Gabor filter bank to generate the corresponding phase map in the myocardium and estimate the location of grid tag intersections. Second, we use a non-rigid registration module driven by thin plate splines (TPS) to generate a transformation function between tag intersections in two consecutive images. Third, deformable spline models are initialized using Fourier domain analysis and tracked during the cardiac cycle using the TPS generated transformation function. The splines will then locally deform under the influence of gradient flow and image phase information. The final motion is decomposed into tangential and normal components corresponding to the local orientation of the heart wall. The new method has been tested on LA phantoms and in vivo heart data, and its performance has been quantitatively validated. The results show that our method can reconstruct the motion field in LA cardiac tagged MR images accurately and efficiently.
\end{abstract}

Keywords: tagged MRI, LA, myocardial motion tracking, Gabor filter, deformable model, TPS.

\section{Introduction}

For decades, heart disease has been the leading cause of death in Western countries [10. Tagged MRI 2] 3] provides a promising approach to the diagnosis and treatment of the disease, by noninvasively both revealing the anatomical structure of the myocardium, and displaying the motion of the myocardium during the cardiac cycle. However, it is still a big challenge to quantitatively track and reconstruct the motion in the living myocardium.

Currently, most tag tracking methods, e.g., Harmonic Phase imaging (HARP) 8], have concentrated on solving the problem of reconstructing the myocardial motion in short axis (SA) images. However, 3D motion analysis including LA image analysis is necessary for a better understanding of the anatomic cause of the heart disease, and to fully evaluate the local heart function. In 9] and [12, motions in multiple SA images have been interpolated to reconstruct the 
motion in 3D. However, these methods did not explicitly address the motion through the SA image plane. In [14, through-plane motion has been encoded as phase information in SA images but, nevertheless, its performance deteriorates at the apex. Moreover, all these methods may generate overly smoothed results because of the small number of SA slices to be interpolated. Therefore, we need to analyze LA images directly, in order to track and reconstruct the myocardial motion in 3D.

The HARP method makes use of the inverse Fourier transform of one harmonic peak of the transform of the MR image in the frequency domain to estimate the local tag phase distribution, and then uses the phase changes over the cardiac cycle to derive the displacement and strain. HARP generates the phase map based on a set of constant global parameters, so it lacks adaptivity to changing local tag spacing and orientation. Its insensitivity to local structure may cause erroneous local phase patterns, such as bifurcations in the phase map. In 13 and [15, responses to a bank of Gabor filters 1] have been used to adaptively detect the local tag spacing and orientation, which are used in turn to estimate the local phase. The phase outputs of Gabor filter banks have high accuracy. However, their performance is limited by the following two factors. First, the performance of Gabor filters degrades near the myocardial boundaries. This is because around the myocardium boundaries, the input to the Gabor filter is a combination of myocardial motion and the independent motion of blood or adjacent issue. Second, the Gabor filters cannot capture large deformations in the myocardium with the same accuracy as for small deformations, as their performance is limited by the size and shape of the filters. Both HARP and the Gabor filter bank method have been implemented for SA image analysis, but there are no current reports of quantitative LA analysis using either method. In [15], Chen et al. used a spline-based scheme to reconstruct the motion of tags through the cardiac cycle. A hybrid of the tag phase and the gradient flow approaches was used to converge spline models and tags. However, the tracking may fail when there is large and nonlinear deformation, since the spline model is initialized as a linear combination of tag locations in previous cardiac phases.

To overcome problems with the existing motion tracking methods, and to find a solution for LA motion analysis, we propose a new motion tracking method. First, two tag phase maps (vertical and horizontal) are generated for grid-tagged MR images, using a Gabor filter bank. The intersections of tags in two consecutive images are nonrigidly registered, using the thin plate spline (TPS) [11] to generate a smooth $2 \mathrm{D}$ transformation function between the pair of images. Each segment of a tag in LA images is modeled as a deformable spline, whose initial location in the current image is decided by previous images and the transformation function. The spline is deformed under the influence of the phase map and local gradients. We then use the virtual spline method in 13 to reconstruct the full $2 \mathrm{D}$ displacement and strain in the LA image.

We give a description to our method in more detail in Section Two. In Section Three, experimental results and validations are presented. We discuss the strengths of our method and draw some conclusions in Section Four. 


\section{Method}

\subsection{Gabor Filter Bank}

A Gabor filter [1] is a sinusoidally modulated Gaussian that can be convolved with an image to extract the local periodic "stripe" content. It is simply expressed in the image domain as a Gaussian multiplied by a complex sinusoid:

$$
h(x, y)=g\left(x^{\prime}, y^{\prime}\right) \cdot \exp \left[-i 2 \pi\left(u_{0} x+v_{0} y\right)\right]
$$

with center frequency $\left(u_{0}, v_{0}\right) ; g\left(x^{\prime}, y^{\prime}\right)=\frac{1}{2 \pi \sigma_{x^{\prime}} \sigma_{y^{\prime}}} \exp \left(-\frac{\left(\frac{x^{\prime}}{\sigma_{x^{\prime}}}\right)^{2}+\left(\frac{y^{\prime}}{\sigma_{y^{\prime}}}\right)^{2}}{2}\right)$ is a Gaussian filter with the spatial standard deviations $\sigma_{x^{\prime}}, \sigma_{y^{\prime}}$. The complex function $h(x, y)$ can be split into its real and imaginary components $h_{R}$ and $h_{I}$ (even and odd functions, respectively), whose Fourier transforms are:

$$
\begin{aligned}
& H_{R}(u, v)=\frac{1}{2}\left(G\left(u-u_{0}, v-v_{0}\right)+G\left(u+u_{0}, v+v_{0}\right)\right) \\
& H_{I}(u, v)=\frac{i}{2}\left(G\left(u-u_{0}, v-v_{0}\right)-G\left(u+u_{0}, v+v_{0}\right)\right)
\end{aligned}
$$

where $G(u, v)$ is the Fourier transformation of $g\left(x^{\prime}, y^{\prime}\right)$, which is also a Gaussian. Therefore the real and imaginary filters in (2) and (3) are the sum of two coupled Gaussian functions in the Fourier domain, centered at the frequencies $\left(u_{0}, v_{0}\right)$ and $\left(-u_{0},-v_{0}\right)$. The final form of a $2 \mathrm{D}$ Gabor filter in the Fourier domain is:

$$
H(u, v)=H_{R}(u, v)+i H_{I}(u, v)
$$

The coupled 2D Gabor filters in the Fourier domain can be parameterized as $\nu, \theta, \sigma$, where $\nu$ is the reciprocal of the tag spacing, $\theta$ is the orientation, and $\sigma$ is the size of the filters. Its response is maximal when the input tagged image has the same local frequency and orientation. During systole, the local spacing (corresponding to $\nu$ ) and orientation (corresponding to $\theta$ ) of tags change and are variably distributed in the myocardium. To find the local frequency and orientation, we use a filter bank, which consists of Gabor filters with 5 different values of $\nu\left(\left[\frac{1}{1.1} \nu_{0}, \frac{1}{1.05} \nu_{0}, \nu_{0}, \frac{1}{0.95} \nu_{0}, \frac{1}{0.9} \nu_{0}\right]\right)$ and $\theta([-\pi / 10,-\pi / 20,0, \pi / 20, \pi / 10]) . \nu_{0}$ is the inverse of the initial tag spacing. The value of $\sigma$ is dependent on $\nu$ to allow a fixed range of output for filters in the bank. Each filter is multiplied with the Fourier transform of the tag image and then undergoes the inverse Fourier transformation to generate a corresponding magnitude image of its output. For each pixel in the tag image, only the three filters that have the largest output at that location in the magnitude image are considered. Parameters of those three filters are nonlinearly interpolated to create an optimal estimation for local frequency (inverse of tag spacing) and orientation at each pixel. The outputs of these three filters are also nonlinearly combined to form a complex image, from which the local phase can be computed using the arctan function. 


\subsection{Motion Tracking}

We track the myocardial motion in two steps. Given two consecutive tag images $I_{i}$ and $I_{i+1}$, we first use a nonrigid registration method to initialize spline models in $I_{i+1}$ based on the tag locations in $I_{i}$, and then use deformable models to improve the tracking.

Nonrigid Registration: During the cardiac cycle, there is motion through the LA image plane, which can cause the appearance and disappearance of tags in the image of the curved heart wall, the papillary muscles may move into or out of the LA image plane, and tags decay as a function of time. All these facts may dramatically change the appearance and shape of the heart in LA images during the cardiac cycle so that it is difficult to register two images directly. To overcome these difficulties, we convert the registration into a problem of matching the corresponding tag intersections in two grid tag images.

Suppose we are tracking the motion of tags from $I_{i}$ to $I_{i+1}$ and assume we have a good segmentation of the myocardium. The tag intersections in the myocardium in these two images are closely correlated except that the throughplane motion may cause the intersections close to the myocardial boundaries to disappear or appear. We denote tag intersections in $I_{i}$ and $I_{i+1}$ as $P=\left\{p_{j}, j=\right.$ $1,2, \ldots, M\}$ and $Q=\left\{q_{k}, k=1,2, \ldots, N\right\}$, respectively. Assume that from $I_{i}$ to $I_{i+1}$, the underlying motion field can be expressed as a non-rigid transformation function $f$. A point $p_{j} \in P$ in $I_{i}$ is mapped to its new location $p_{j}^{\prime}=f\left(p_{j}\right)$ in $I_{i+1}$. Thus the matching problem is equivalent to the minimization of fuzzy assignment-least square energy function $E_{Y, f}$ :

$E(Y, f)=\sum_{j=1}^{M} \sum_{k=1}^{N} y_{j k}\left\|q_{k}-f\left(p_{j}\right)\right\|^{2}+\lambda\left\|f^{\prime}\right\|^{2}+T \sum_{j=1}^{M} \sum_{k=1}^{N} y_{j k} \log y_{j k}-\zeta \sum_{j=1}^{M} \sum_{k=1}^{N} y_{j k}$

$\lambda$ and $\zeta$ are both positive weights for terms in the energy function, $\lambda$ controls the strength of the smoothness constraint, and $\zeta$ controls the strength of the internal correlations between points. $Y$ is the correlation matrix between two point sets. Its element $y_{j k}=\frac{1}{T} \exp \left(\frac{-\left\|q_{k}-p_{j}^{\prime}\right\|^{2}}{2 T}\right)$ satisfies the constraint $\sum_{j=1}^{M+1} y_{j k}=1$ for $k=1,2, \ldots, N$ and $\sum_{k=1}^{N+1} y_{j k}=1$ for $j=1,2, \ldots, M$ with $y_{j k} \in[0,1]$. Also, an entropy term, $T \sum_{j=1}^{M} \sum_{k=1}^{N} y_{j k} \log y_{j k}$, is added to the energy function, following the approach of deterministic annealing [6]. The temperature parameter $T$ is higher at the start of the tracking process so that the energy function favors fuzzy correspondence to maintain its convexity, and gradually decreases to zero during the tracking for a global binary solution of $Y$. The second term is a smoothness constraint on the transformation function $f$, and the fourth term is used to control the existence of outliers in the final matching result. Notice the first two terms in the function are in the form of a thin plate spline (TPS) function and $f$ can be solved for using the QR decomposition [4. By iteratively updating $Y$ and $f$, we can solve for $f$ in the form of a combination of the global translation matrix $t$, and local deformation vertices $w$ at tag intersections. The 
local deformation vertices will be smoothly interpolated to generate a $2 \mathrm{D}$ local deformation map for each pixel in $I_{i}$. Given the location of tags in $I_{i}$, their corresponding locations in $I_{i+1}$ can be estimated using the global translation followed by the local deformation.

Deformable Models: We initialize spline models at the locations computed using the non-rigid registration. We initialize independent splines for each segment of a tag so that the motions in the septum and free wall will not interfere with each other. The contours move under the influence of the external gradient force $f_{\text {ext }}$ and the phase constraint $f_{\text {phase }}$, following the Lagrangian equation:

$$
\dot{d}+\mathbf{K} d=f_{\text {ext }}+f_{\text {phase }}
$$

where $\mathbf{K}$ is the stiffness matrix that controls the smoothness of the deformable contour [7, $d$ is the displacement corresponding to the initial locations of spline models (after the nonrigid registration at each cardiac phase), and $\dot{d}$ is the speed of the deformation. The external force is derived from the tagged MRI image, in the form of gradient flow:

$$
\left.f_{\text {ext }}=-\nabla(g \cdot I)\right)
$$

where $g$ is the Gaussian operator, and $I$ is the original image. The external force alone cannot guarantee an accurate convergence of the deformable contour and the corresponding tag because of local noise, imaging artifacts, and small tag spacing. Therefore we design a supplementary phase force field, which is also derived from the output of the Gabor filter bank, to constrain the movement of deformable splines. Given the wrapped phase output $R$ of the Gabor filter bank, the phase constraint at pixel $x \in I_{i+1}$ has the following form:

$$
f_{\text {phase }}(x)=-\left|U\left(R_{i}(x)-R_{i+1}(x)\right)\right|^{-1} \cdot \nabla(\pi-|R(x)|)
$$

where $U(\cdot)$ is an unwrapping operator. The magnitude of the phase constraint is inversely proportional to the local phase change so that the phase influence decreases when the local deformation increases, and vice versa.

\subsection{Displacement and Strain Reconstruction}

The shape and motion pattern of the myocardium in LA cardiac images are different from those in SA images. In SA images, the myocardium around the left ventricle (LV) has an annulus shape and the motion of the myocardium can be described as a combination of radial stretching (pointing towards the centroid of the LV) and circumferential shortening. In LA images, the myocardium around the LV has the shape of a horseshoe and the motion cannot readily be divided into radial and circumferential components. Therefore we calculate the principal strains, P1 and P2, which are usually coincident with the normal and tangential components of the local heart wall orientation, respectively.

After spline models converge to follow the tags, we use the virtual tag methods [13] to generate a 2D displacement map. A smoothness constraint is used where there are not enough tags to be reliably interpolated. The strain is then calculated using the algorithm introduced in [5]. 


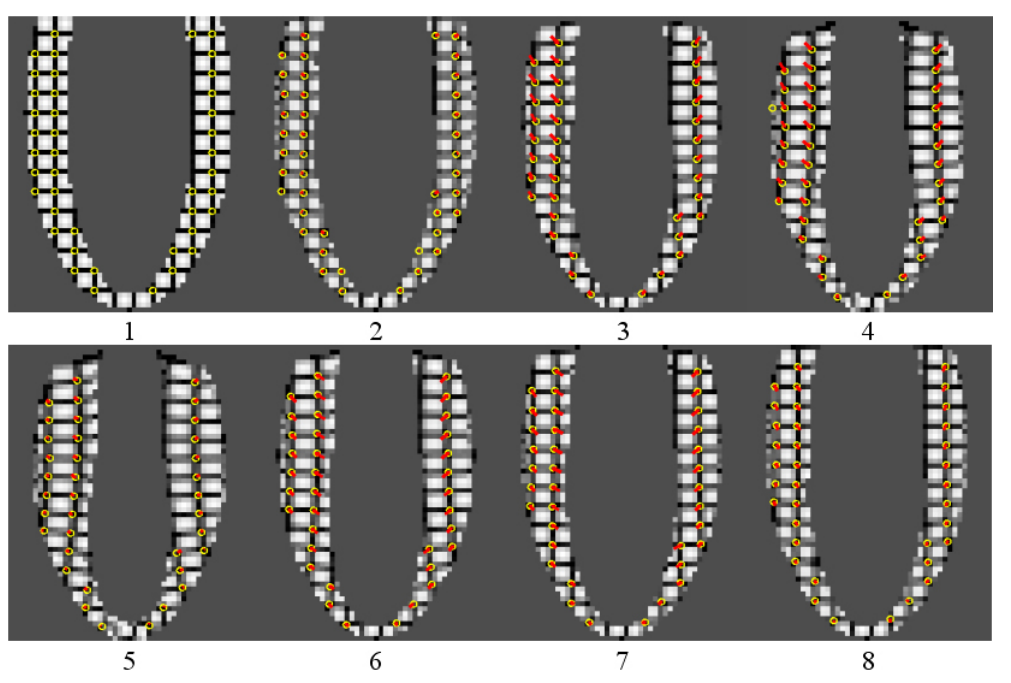

Fig. 1. The TPS-driven non-rigid point matching in the numerical phantom from phase 1 to 8 . Yellow circles are tag intersections to be matched. The red arrows show the direction of the transformation function. At phase 4 there is an erroneous intersection, which is discarded in the following phase since there is no correspondence.

\section{$3 \quad$ Experimental Results}

We first tracked the motion in a numerical phantom using our tag tracking method. The phantom deforms under a given displacement field. The LV in LA images is modeled as two co-focus ellipsoids. The phantom is thinner at the apex to resemble the anatomic structure of the myocardium. The motion in the myocardium is originally defined in directions that are tangential and normal to the local orientation of the phantom, and then transformed into $x-$ and $y-$ displacements in the Cartesian coordinates. At the starting cardiac phase, tags are initialized as straight dark lines in the phantom, using a sine function for the tag intensity profile. the tags fade during the simulated cardiac cycle. Two Gabor filter banks are used to generate tag phase maps in the $x$ and $y$ directions. The intersections of tags are defined as local minima in both phase maps. The nonrigid registration tool is used to find the correspondence between intersections in two images and generate a transformation function. In Fig. 1 we show the motion tracking process.

The final displacement map is calculated using the deformable model. In Fig. 2 we display the $x$ - displacement map at the simulated cardiac phases 2,3 , and 4. We can see the displacement has been well captured qualitatively. To quantitatively validate the tracking performance of our method, we also calculated the root mean square (RMS) error in the computed displacement map during the simulated cardiac cycle. The result is shown in Tab. 1. The mean magnitude of the "ground truth" $x$ - displacement map is compared with the RMS error, 


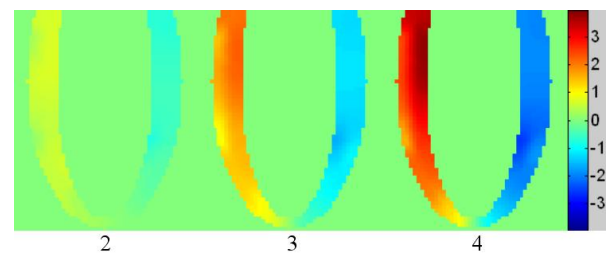

Fig. 2. The $x$ - displacement map of the phantom at cardiac phases 2,3 , and 4

Table 1. The comparison between the RMS error and the mean magnitude of the $x-$ displacement (both in pixels)

\begin{tabular}{|c|c|c|c|c|c|c|c|c|c|c|}
\hline phase & 1 & 2 & 3 & 4 & 5 & 6 & 7 & 8 & 9 & 10 \\
\hline Mean Disp. & 0.3 & 0.9 & 1.5 & 1.8 & 1.2 & 0.6 & 0.36 & 0.24 & 0.12 & 0.06 \\
\hline RMS error & 0.0401 & 0.1013 & 0.1511 & 0.1768 & 0.1411 & 0.1230 & 0.0615 & 0.0303 & 0.0297 & .0156 \\
\hline Ratio (\%) & 13.37 & 11.26 & 10.07 & 9.82 & 11.76 & 20.50 & 17.08 & 12.63 & 24.75 & 26.00 \\
\hline
\end{tabular}

both in pixels. Notice in the first 5 phases, the RMS errors are below $15 \%$ of the corresponding mean displacement. However, in the last 5 phases the error ratios increase. This can be explained by the accumulative error in deformable model fitting, and the decreasing contrast between tagged and untagged regions in the phantom (to simulate the decay of tags).

We tested our method on six in vivo heart data sets. In Fig. 3, we show the result of tracking of myocardial motion and the corresponding strain distribution of a patient with hypertrophic cardiomyopathy. The total processing time for one image sequence (usually including 10100 by 100 frames) on a PC with $2 \mathrm{GHz}$ $\mathrm{CPU}$ is within one minute.
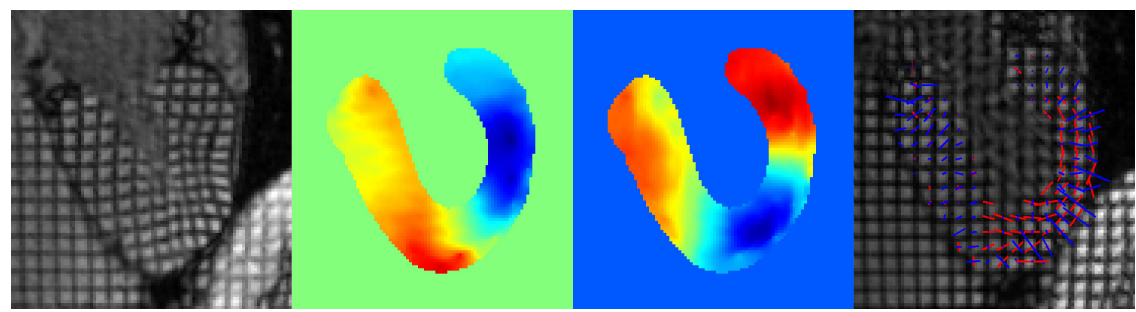

Fig. 3. From left to right, the deformed tagged MR image; $x$ - displacement, maximal $x$ - displacement 6.113 pixel, minimal $x$ - displacement -7.2496 pixel; $y$ - displacement, maximal $y$-displacement 7.1756 pixel, minimal $y$ - displacement -1.6411 pixel; and the P1 and P2 strain map projected onto the undeformed image, in which red lines show the magnitude and orientation of P1 strain, and blue lines represents the magnitude and orientation of P2 strain. It is clear that the free wall and the septum have different strain distributions that may be of clinical importance. 


\section{Conclusions}

We propose in this paper a new motion tracking method for the analysis of myocardial motion in LA images. Experimental motion tracking results of both the phantom and the in vivo heart data demonstrate the method's effectiveness. More experiments are necessary for finding its optimal performance point. The motion tracking method can also be used in the analysis of SA images. In the future, we will extend the method into a true 3D motion analysis tool for use in clinical studies. In particular, we hope to collect enough in vivo data with large LV deformation, where 3D motion may be critical for pathological analysis. Some preliminary work along these lines, including the development of a TPS-based 3D tracking module, has been started.

\section{References}

1. Gabor, D.: Theory of communication. J. IEE 93(3), 429-457 (1946)

2. Axel, L., Dougherty, L.: MR imaging of motion with spatial modulation of magnetization. Radiology 171, 841-845 (1989)

3. Axel, L., Dougherty, L.: Improved method of spatial modulation of magnetization (SPAMM) for MRI of heart wall motion. Radiology 172, 349-350 (1989)

4. Wahba, G.: Spline models for observational data. SIAM, Philadelphia, PA (1990)

5. Fung, Y.C.: Biomechanics: Mechanical Properties of Living Tissues, 2nd edn. Springer Science, New York (1993)

6. Gold, S., Rangarajan, A.: A graduated assignment algorithm for graph matching. IEEE Trans. Pattern Analysis and Machine Intelligence 18(4), 377-388 (1996)

7. Metaxas, D.: Physics-based Deformable Models: Application to Computer Vision, Graphics and Medical Imaging. Springer, Heidelberg (1996)

8. Osman, N.F., McVeigh, E.R., Prince, J.L.: Imaging heart motion using Harmonic Phase MRI. IEEE Trans. on Medical Imaging 19(3), 186-202 (2000)

9. Amini, A.A., Chen, Y., Elayyadi, M., Radeva, P.: Tag surface reconstruction and tracking of myocardial beads from SPAMM-MRI with parametric B-spline surfaces. IEEE Trans. on Medical Imaging 20(2), 94-103 (2001)

10. American heart association 2002 annual report, by American Heart Association (2002)

11. Chui, H., Rangarajan, A.: A new point matching algorithm for non-rigid registration. Computer Vision and Image Understanding 89(2-3), 114-141 (2003)

12. Chang, H., Moura, J.M.F., Wu, Y., Sato, K., Ho, C.: Reconstruction of 3D dense cardiac motion from tagged MR sequences. In: Proceedings of ISBI, pp. 880-883 (2004)

13. Axel, L., Chen, T., Manglik, T.: Dense myocardium deformation estimation for 2D tagged MRI. In: Frangi, A.F., Radeva, P.I., Santos, A., Hernandez, M. (eds.) FIMH 2005. LNCS, vol. 3504, pp. 446-456. Springer, Heidelberg (2005)

14. Abd-Elmoniem, K.Z., Stuber, M., Osman, N.F., Prince, J.L.: ZHARP: Threedimensional motion tracking from a single image plane. In: Christensen, G.E., Sonka, M. (eds.) IPMI 2005. LNCS, vol. 3565, pp. 639-651. Springer, Heidelberg (2005)

15. Chen, T., Axel, L.: Using Gabor filters bank and temporal-spatial constraints to compute 3D myocardium strain. In: Proceedings of EMBC (2006) 